\title{
Influence of Transitional Metals Doping on Conductivity of Collagen Lyophilisate and Elastin
}

\author{
M. GauZA* AND L. Kubisz
}

Department of Biophysics, Poznań University of Medical Sciences, A. Fredry 10, 61-701 Poznań, Poland

\begin{abstract}
Knowing that conductivity is a temperature function $\sigma=f(T)$ allows us to estimate the thermal stability of collagen preparations from fish skin. Measurements can provide information, which is crucial for technologies applying this type of collagen. Conductivity $\sigma$ measurements were performed in a constant electric field $E=1 \mathrm{kV} / \mathrm{m}$, in the temperature range of $290-480 \mathrm{~K}$. White collagen lyophilisate was used as control material. It contained neither elastin nor melanin dopants nor non-organic dopants such as gold, copper, silver or zinc. Gold, copper, silver and zinc belong to the group of transitional metals and they have intermediate properties of both alkaline metals and metals in the $p$ block of the periodic table. The common feature of most of these elements is their ability to form coordination complexes of intense color. In the measured materials peaks were found in the temperature range of 303-323 K. Peak temperature and its shape depend on the type of material. In the case of nano-gold doping a drop in peak temperature and a decrease in conductivity were observed. The measured materials are characterized by lower thermal resistance than bovine collagen. Nevertheless, thermal stability of fish skin collagen is high enough for fish skin collagen to replace bovine collagen. The measurement results obtained for white collagen and white collagen with $\mathrm{Zn}^{2+}$ may indicate better bonding of free water. The presence of nano-silver and nano-copper as doping agents in graphite collagen may lead to a decrease in hygroscopy of the material and, as a result, may increase its resistance to bacteria.
\end{abstract}

PACS: 72.20.-i, 72.80.Le

\section{Introduction}

Cross-linking in fish skin collagen is not as good as in bovine and swine skin collagen. As a result, solubility of fish skin collagen is higher than solubility of other types of collagen. Furthermore, higher solubility of fish skin collagen allows to obtain a native form of collagen in the final stage - a collagen hydrogel. This is possible when applying a sensitive method of receiving this protein under conservative conditions $[1,2]$. Collagen can be also obtained in the form of lyophilisate.

Lyophilisation is the process of sublimation drying. It is used in thermally stable products (non-resistant to heating) such as pharmaceuticals and cosmetics or foods. Lyophilized collagen includes about $98 \%$ of collagen, which can be used for making cosmetics and pharmaceuticals. As a result of lyophilisation the substance keeps its spatial layout of amino acids and is biologically active. Collagen is an element of fibrous connective tissue. Cell components of connective tissue take part in building and regenerative processes of connective tissue. Specialized cells of packed connective tissue are capable of, among other things, producing collagen, which is able to bond salts of some metals. Since change in electrical conductivity is a temperature function $\sigma=f(T)$, it allows to estimate thermal stability of collagen materials from fish skin. Measurements of temperature dependence of electrical conductivity can provide information, which is vital from the point of view of technologies applying fish skin collagen.

\footnotetext{
* corresponding author; e-mail: mgauza@amp.edu.pl
}

\section{Materials}

The material used in the experiment was fish skin collagen (FS - fish skin; family: Cyprinidae) obtained by means of sour hydration process $[1,2]$. For measurements collagen lyophilisates with different amount of melanin were used. The colour of lyophilisate gives evidence of its content. White collagen contains less melanin than graphite one. Furthermore, collagen used in the measurements was doped with gold, copper, silver and zinc (Table).

Contents of metal dopants in collagen.

TABLE

\begin{tabular}{c|c|c}
\hline \hline $\begin{array}{c}\text { Doping } \\
\text { type }\end{array}$ & Material & $\begin{array}{c}\text { Doping } \\
\text { concentration }\end{array}$ \\
\hline silver & in graphite collagen & $1.92 \mathrm{mg} \mathrm{Ag} / 1$ \\
copper & & $1.92 \mathrm{mg} \mathrm{Cu} / 1$ \\
gold & & $1.92 \mathrm{mg} \mathrm{Au} / 1$ \\
zinc & & $2.38 \mathrm{mg} \mathrm{Zn} / 1$ \\
zinc & in white collagen & $2.18 \mathrm{mg} \mathrm{Zn} / 1$
\end{tabular}

Cylindrical samples, $10 \mathrm{~mm}$ in diameter and 0.30 $0.05 \mathrm{~mm}$ to $1.00 \pm 0.05 \mathrm{~mm}$ thick made of $2 \mathrm{MPa}$ lyophilisated collagen compressed under pressure were used for the measurements. A specially constructed device sustained a complex geometry of the sample. Sample thickness was measured using a micrometer. Silver paste electrodes and thin copper wires were attached to the sample. The copper wires connected the sample to the measuring circuit. 
The sample prepared in such a way was put in a specially designed hermetic measuring frame in controlled temperature. The heating rate was $2 \mathrm{~K} / \mathrm{min}$ and a temperature regulator sustained it. Sample temperature was measured by a constantan-copper thermocouple, irrespective of the Pt100 sensor indications. The sensor worked within the feedback loop. One of the thermocouple ends was placed within $1-2 \mathrm{~mm}$.

\section{Methods}

Electrical conductivity measurements $\sigma$ were carried out in the temperature range of $290-480 \mathrm{~K}$, in a constant electric field $E=1 \mathrm{kV} / \mathrm{m}$ which guaranteed application of the Ohm law. The value has been established earlier [3]. Because of a high electrical resistance of the measured material, an electrometer was used to measure electric current intensity. The values for current intensity and thermocouple voltage were recorded by a computer. Relative error of electrical conductivity was less than $\pm 5 \%$.

Measurements of temperature dependence of electric conductivity were conducted using the multiple heating method. The method has been adopted from thermally stimulated depolarization current (TSDC) technique [4].

Measured material is heated above its characteristic thermal stability point, that is, above the peak temperature or above the temperature of the inflection point. In the case of collagen these points are related to the processes of thermal denaturation and vitrification, respectively. The sample was kept for a certain time in a temperature a few degrees higher than the temperature of the characteristic point. Next, it was cooled down to its initial temperature and heated again to its next characteristic point. This way of heating is supposed to check reversibility of the observed process. If the process is irreversible, an earlier recorded characteristic point disappears.

The samples were heated to $377 \mathrm{~K}$, and kept in this temperature for about $30 \mathrm{~min}$. Next, they were cooled down to $296 \mathrm{~K}$ and heated again to $440 \mathrm{~K}$. The aim of heating to $377 \mathrm{~K}$ (first heating) was to detect changes connected with releasing free and bound waters. The aim of the second heating to $440 \mathrm{~K}$ was to observe the process of thermal denaturation of dry fish collagen in this temperature [5].

\section{Results and discussion}

Figures 1 and 2 show the electric conductivitytemperature relation recorded for particular materials.

Earlier results show that the size of peak observed in the temperature range of about $320-350 \mathrm{~K}$ is correlated with air humidity [6]. Heating in the temperature of $377 \mathrm{~K}$ is supposed to remove bound water. Bound water can only be released below the temperature of $383 \mathrm{~K}$. Before being evaporated the water transits into free water. Heating to the temperature of $440 \mathrm{~K}$ was supposed

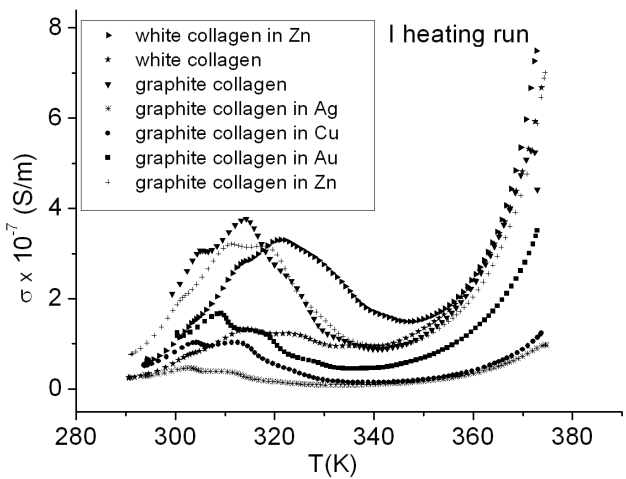

Fig. 1. The electric conductivity-temperature relation in the 1st round of collagen lyophilisates heating.

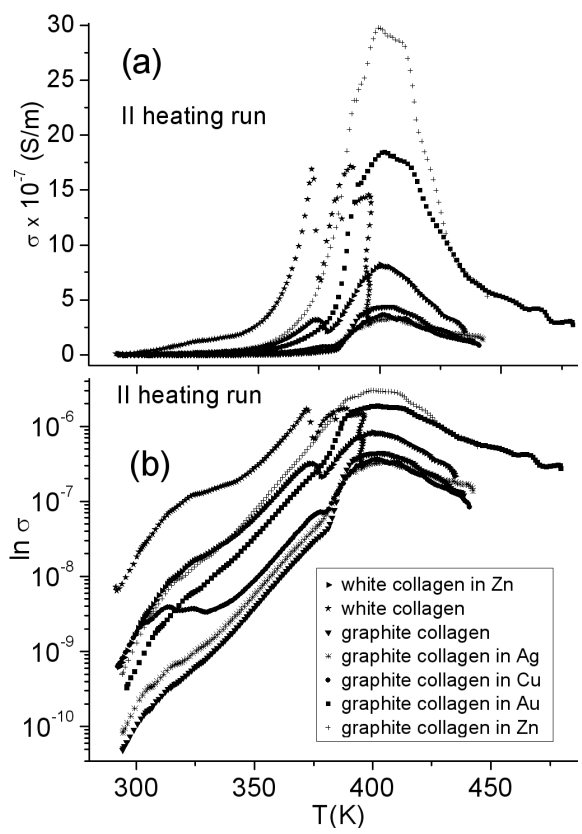

Fig. 2. The electric conductivity-temperature relation in the 2nd round of collagen lyophilisate heating: (a) linear coordinate system; (b) semilogarithmic coordinate system.

to cause a phase change of the material, which is part of the thermal denaturation process.

The results obtained during heating from $295 \mathrm{~K}$ to $373 \mathrm{~K}$ are collected in Fig. 1. White collagen lyophilisate was a control material since it was not organically doped with elastin or melanin, which affects the color. Neither was the collagen doped with non-organic particles such as gold, copper, silver or zinc.

For all the measured materials a peak was found in lower temperatures, that is $303-323 \mathrm{~K}$. Peak temperature and shape depended on the material. Furthermore, a curve deformation could be noticed in the temperature of about $320 \mathrm{~K}$. The deformation resembled an inflection point and there is a high probability that this was a hidden peak. 
Further sample heating resulted in minimum electrical conductivity of collagen in the temperature of about $335-350 \mathrm{~K}$. When heating up to $370 \mathrm{~K}$ an exponential growth could be observed. Most probably this resulted from eliminating bound water.

When comparing dependences of electrical conductivity on temperature for white collagen lyophilisates, it can be noticed that melanin doping, which gives the collagen its color, results in a decrease of electrical conductivity of the material. Furthermore, it results in peak blurring and a decrease of its temperature. What this may mean is that the process of free water loss is facilitated.

When comparing graphite collagen doped with nano-gold with graphite collagen, it could be noticed that nano-gold doping resulted in peak temperature decrease as well as a decrease in its electrical conductivity. Nano-silver and nano-copper doping of graphite collagen resulted in two nonsignificant peaks in this temperature range.

This may mean that since free water could not bound freely, its amount decreased. For example, polar regions on the surface of a collagen molecule, which typically bond water, were blocked. In both cases it is possible to decrease hygroscopy of the material, which may result in a better resistance to bacteria.

Figure 2 presents $\sigma=f(T)$ dependence for the measured materials (without free water, but with trace quantities of bound water) in the second heating. Because of a significant range of variability of electrical conductivity the results are shown both in a linear coordinate system as well as semi-logarithmic coordinate system.

The results shown in Fig. 2a indicate an increase in electrical conductivity resulting from temperature increase in all the measured materials. For most of the materials peak was observed in the temperature of $400 \mathrm{~K}$, which may result from the thermal denaturation process. Visual assessment of the samples heated to $440 \mathrm{~K}$ (distinct darkening of samples) and higher allows to state that the denaturation process is connected with material oxidation, the initial state of oxygen pyrolysis. This means a significantly lower thermal stability of the measured material when compared with bovine material. Graphite collagen doped with $\mathrm{Zn}^{2+}$ ions can be heated to the temperature of $450 \mathrm{~K}$ with no changes to its structure.

Data analysis presented in Fig. 2b leads to the conclusion that the process of free water releasing did not finish in the first round of heating. The presence of an insignificant peak in the temperature range of about $305-330 \mathrm{~K}$ may indicate this fact. Therefore, a peak was observed in the temperature characteristic of free water release, that is, at about $315 \mathrm{~K}$.

Location of this peak depends on the type of material. In the case of collagen doped with nano-copper, especially a significant residual peak in the temperature of about $315 \mathrm{~K}$ should be noticed.

The measurements performed confirmed that knowing the character of changes in electrical conductivity as a temperature function $\sigma=f(T)$ allows to estimate thermal stability of materials from fish skin collagen. The measured materials are characterized by lower thermal resistance than bovine collagen. Nevertheless, the thermal stability of fish skin collagen is high enough to replace bovine collagen. It is also worth pointing out that bovine and fish skin collagen behave similarly in the temperature range of $295-340 \mathrm{~K}$.

Measurement results obtained for white collagen and white collagen doped with $\mathrm{Zn}^{2+}$ may indicate better bonding of free water, which is important in the case of anti-aging cosmetics. Nano-silver and nano-copper doping of graphite collagen may result in lower hygroscopy of the material and increase its resistance to bacteria.

The obtained results provide a wider spectrum of possible applications of the investigated material.

\section{Conclusions}

A characteristic feature of white collagen up to the denaturation temperature is its electrical conductivity, which is higher than in the case of other materials.

Doping did not influence denaturation process, which was indicated by an almost exact peak for all the measured materials at the temperature of about $400 \mathrm{~K}$.

Because the peak size depends on the amount of free water, gold, silver and copper doping of graphite collagen resulted in a decreased conductivity in the temperature range of $302-323 \mathrm{~K}$. This can be seen as a decrease in hygroscopy of the material. In practice this can lead to better resistance to bacteria.

An increase of melanin in collagen increases its conductivity. Peak broadening and its temperature decrease may be related to easier loss of free water than it is in the case of white collagen.

Fish skin collagen behaves similar to bovine collagen in the temperature range of $295 \mathrm{~K}$ to $340 \mathrm{~K}$. The measurements performed indicate that in the temperature range up to $340 \mathrm{~K}$, bovine achilles tendon (BAT) collagen can be replaced by FSC collagen.

\section{References}

[1] J.E. Przybylski, K. Siemaszko-Przybylska, Patent 190737, Urząd Patentu RP, 2002.

[2] J.E. Przybylski, Patent US 7285638, B2 (2007).

[3] M. Gauza, L. Kubisz, Acta Phys. Pol. A 118, 54 (2010).

[4] J. Vanderschueren, J. Gasiot, Topics Appl. Phys. 37, 135 (1979).

[5] M. Gauza, L. Kubisz, Influence of Temperature on Electrical Conductivity of Fish Skin Collagen, Some Aspects of Medical Physics - in vivo and in vitro, Eds. Z. Drzyzga, K. Ślosarek, HARD Publ. Co., Olsztyn 2010, p. 101.

[6] M. Gauza, L. Kubisz, J. Non-Cryst. Solids 357, 686 (2011). 